\title{
Attention orienting by eye gaze and arrows reveals flexibility to environmental changes.
}

\author{
AUTHOR(S): \\ Zhao, Shuo; Uono, Shota; Yoshimura, Sayaka; \\ Toichi, Motomi
}

\section{CITATION:}

Zhao, Shuo ...[et al]. Attention orienting by eye gaze and arrows reveals flexibility to environmental changes.. Acta psychologica 2014, 150: 100105

\section{ISSUE DATE:}

2014-07

URL:

http://hdl.handle.net/2433/189079

\section{RIGHT:}

(C) 2014 Elsevier B.V.; This is not the published version. Please cite only the published version.; この論文は出版社版でありません。引用の際に は出版社版をご確認ご利用ください。 


\section{Attention orienting by eye gaze and arrows reveals flexibility to environment changes}

Shuo Zhao ${ }^{1,3}$, Shota Uono ${ }^{1}$, Sayaka Yoshimura $^{2}$, Motomi Toichi ${ }^{1,3}$

${ }^{1}$ Faculty of Human Health Science,

${ }^{2}$ Department of Psychiatry,

Graduate School of Medicine, Kyoto University, Kyoto, Japan

${ }^{3}$ Organization for Promoting Developmental Disorder Research, Kyoto, Japan

Correspondence concerning this article should be addressed to Shuo Zhao, Faculty of

Human Health Science, Graduate School of Medicine, Kyoto University, 53 Shogoin

Kawahara-cho, Sakyo-ku, Kyoto 606-8507, Japan.

Tel: +81 - $75-751-3966 ;$ FAX: +81 - $75-751-3966$;

E-mail: zhaoshuo09@gmail.com 


\section{Abstract}

This study aimed to evaluate the difference in non-predictive cues between gaze and arrows in attention orienting. Attention orienting was investigated with gaze or arrows as separate cues in a simple condition (i.e., block design) in Experiment 1 and in an unpredictable condition (i.e., randomized design) in Experiment 2. Two kinds of sound (voice and tone) stimuli were used as targets. Results showed that gaze and arrow cues induced enhanced attention orienting to a voice versus tone target in the block condition. However, in the randomized condition, enhanced attention orienting to a voice versus tone target was found in gaze but not arrow cues. The congruency of the meaning between a social cue (i.e., gaze) and a social target (i.e., voice) was clear in the randomized but not blocked design, because social gaze and non-social arrow cues were implemented in the same block. Thus, attention orienting might be mediated by the associated relationship of cue-target in a randomized condition, as an enhanced orienting effect was found when the associated relationship of cue-target was strong (i.e., social cue and target). The present study suggests that the difference in attention orienting between gaze and arrows is apparent in a randomized design (the unpredictable condition), and people employ a flexibly strategy of orienting to better respond to environmental changes. 


\section{Introduction}

Attention orienting plays an important role in our everyday life, and a variety of cues are used as triggers of attentional orientation. For example, social signs such as eye gaze reflect a uniquely human ability underlying social communication. It would help us to better identify a person's focus and understand their thoughts, intentions, beliefs, and desires, when observing their gaze during human interactions (Baron-Cohen, 1995). Similar to social signs, non-social signs also influence our attention orienting every day, such as an arrow on a road sign. Nevertheless, compared with eye gaze, an arrow does not have the social immediacy of a person looking or sharing interactions.

In the past several years, many studies have focused on comparing the role of directional gaze and arrows in attention orienting. These studies commonly investigated attention orienting by gaze and arrows using the modified version of Posner's cueing paradigm (Posner, 1980). For example, Friesen \& Kingstone (1998) presented uninformative gazes as cues (directed toward right or left), indicating the location of the target (appearing on the right or left). The reaction times (RTs) to detect a target are faster when the target appears in the same direction as the cues than in the opposite direction. 
Most of these studies provided evidence of similar shifts of attention orienting when an arrow instead of a gaze was used as the cue (Bayliss \& Tipper, 2005; Borjon, Shepherd, Todorov, \& Ghazanfar, 2011; Kuhn \& Kingstone, 2009; Tipples, 2002). Contrary to these findings, some researchers have proposed that attention orienting was different between gaze and arrows. A previous study (Marotta, Lupiáñez, Martella, \& Casagrande, 2012) found that an object-based effect occurred only when an arrow was presented as a cue to orienting, whereas a location-based effect only occurred with eye gaze as the cue. Furthermore, another recent study (Friesen, Ristic, \& Kingstone, 2004) using the counterpredictive paradigm (the target was more likely to appear in the location opposite the one indicated by the cue) found that reflexive orienting shifts to the cued locations were only observed when using gaze as the cue. These studies only implemented gaze and arrows as cues, separately, in a simple environment (i.e., block condition). In real life, however, eye gaze and arrows are often mixed, and therefore a different division of roles may occur in a complex environment, which is related to the ability of how to rapidly respond to danger. Therefore, it is also important to investigate attention orienting between gaze and arrow cues when they are embedded in a rich environment, which is suggested to be the only apparent difference between them (Birmingham \& Kingstone, 2009). 
Previous studies have reported that attention orienting is influenced by target processing. Some studies reported that joint attention occurred using interest-related materials following training in subjects with autism spectrum disorder who have impaired joint attention (Kryzak, Bauer, Jones, \& Sturmey, 2013; Naoi, Tsuchiya, Yamamoto, \& Nakamura, 2008). Another study found an enhanced effect of attention orienting when used a social stimulus as the target; that is, a greater effect of orienting was found when the target appeared on a face than a scrambled face in participants with low autistic-like traits (Bayliss \& Tipper, 2005). Additionally, in our study (Zhao et al., 2013), an associated relationship between cue and target was proposed to explain a similar enhanced orienting effect when using sounds as the targets. Greater gaze-triggered orienting was found when voice was the target rather than tone, indicating that a strongly associated relationship between gaze and voice may elicit an enhanced effect on attention orienting. However, it is still not understood whether the associated relationship of cue-target can also be used with arrows as cues, and it may be possible to reflect different attention orienting between gaze and arrows when they are mixed in a rich environment.

In the present study, we examined attention orienting with gaze and arrows as cues using Posner's cueing paradigm under a block design (simple condition) 
(Experiment 1) and a randomized design (unpredictable condition) (Experiment 2). In addition, we manipulated two sounds as targets, i.e., social voice and non-social tone, to refer the associated relationship between the cue and target. The aims of the study were, (1) to investigate whether attention orienting between gaze and arrows as cues could be distinguished by the associated relationship of cue-target and (2) to examine whether attention orienting between gaze and arrows as cues might only become apparent under the unpredictable condition, which seems closer to a real environment than the simple condition.

\section{Experiment 1}

In Experiment 1, we manipulated attention orienting between gaze and arrow cues by two types of sounds as targets (voice and tone) under a block condition.

\subsection{Materials and Methods}

\subsubsection{Ethics Statement}

The experimental procedures were approved by the local ethics committee of Kyoto University Graduate School and Faculty of Medicine and were carried out in accordance with the principles of the 1964 Declaration of Helsinki. 


\subsubsection{Participants}

Twenty-seven naïve participants (mean age $=21.44 \pm 2.56$ SD years; 14 men) took part in the study and received payment. They were recruited from Kyoto University. All participants were right-handed, as assessed by the Edinburgh Handedness Inventory (Oldfield, 1971), and had normal or corrected-to-normal visual and auditory acuity.

\subsubsection{Design}

The order of cueing paradigms (gaze/arrow) was presented in two blocks, which were counterbalanced across participants. Each block had a two-factor repeated measure design. Auditory targets had two levels: voice and tone. Validity had two levels: valid trials (direction of the cue was congruent with sound target location) and invalid trials (direction of the cue was incongruent with sound target location).

\subsubsection{Stimuli}

In the gaze cue block (Figure 1A), we selected the cue stimuli from Ekman and Friesen (1976). Photographs of a female model with a neutral face were selected. The gaze direction was then manipulated. The irises and pupils of the eyes were cut from the original photographs and pasted to fit over 18 pixels for the right or left side of the eyes using Photoshop 5.0 (Adobe). We cropped the photographs in an ellipse $8.3^{\circ}$ wide and $12.1^{\circ}$ high to exclude the hair and background. In the arrow cue block (Figure 1B), a 
symmetrical arrow was presented as cue stimuli, with an arrowhead at one end and the trial at the opposite end. The arrows measured $8.3^{\circ}$ in width by $3.0^{\circ}$ in height and were light gray.

In addition, two types of auditory stimuli were presented as targets. One was sampled from a native Japanese woman; an /i/ voice sound (F0 frequency of 300Hz, $80 \mathrm{~dB}$ SPL), which is similar to the /iy/ sound in English. The other was a pure tone of similar frequency to the F0 voice $(300 \mathrm{~Hz}, 80 \mathrm{~dB}$ SPL), which was produced with the Audacity V1.3.13 (AudacityStore.com). The duration of the stimulus presentation was $150 \mathrm{~ms}$.

\subsubsection{Apparatus}

Stimulus presentation and data acquisition were controlled by Presentation (Neurobehavioral Systems) on a Windows computer. Stimuli were presented on a 19-inch monitor (Dell: screen resolution $1024 \times 768$ pixels; refresh rate $60 \mathrm{~Hz}$ ). The distance between the monitor and the participants was fixed at approximately $57 \mathrm{~cm}$ using a headrest. All of the auditory stimuli were presented through headphones. The response time (RT) measurements were based on a response button.

\subsubsection{Procedure}


The sequence of stimulus presentation is shown in Figure. 1. For each trial, a fixation cross point was first presented for $600 \mathrm{~ms}$ in the center of the screen. A neutral stimulus of straight eye gaze (gaze block) or transverse lines (arrow block) was then presented at the location. After 500ms, a cue stimulus (e.g., gaze or arrow) of right or left direction was presented in the center of the screen. The stimulus onset asynchrony (SOA) between the auditory target and cue was manipulated for $200 \mathrm{~ms}$. Subsequently, an auditory stimuli target (voice or tone sound) was presented in the left or right ear for $150 \mathrm{~ms}$ through headphones. The participants were asked to respond as quickly and exactly as possible whether the target appeared on the left or right side of the screen by pressing the corresponding key on the switch keypad using their dominant index or middle finger, respectively. Response times (RTs) were measured in each trial. The cue display remained until the response or until 2150ms had elapsed. The targets appeared randomly on the same or opposite side to the gaze direction when the eyes looked left or right. The target appeared at the cued location in $50 \%$ of the trials. The participants were told that the cue did not predict the target location and were instructed to fixate on the center of the screen in each trial. 
A
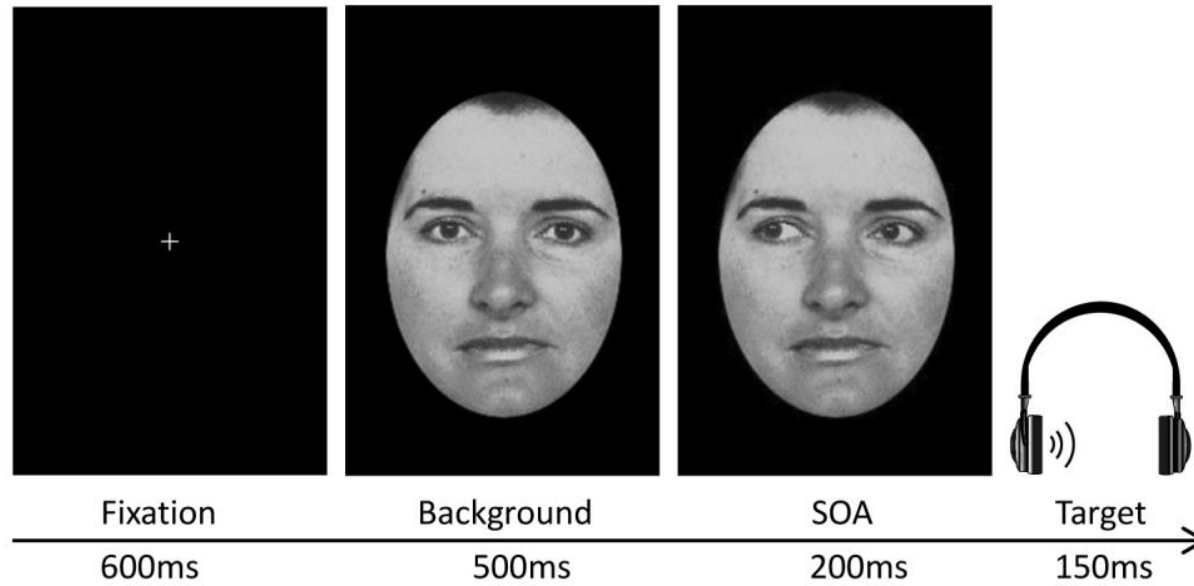

Background

SOA $\underset{150 \mathrm{~ms}}{\text { Target }} \rightarrow$

B
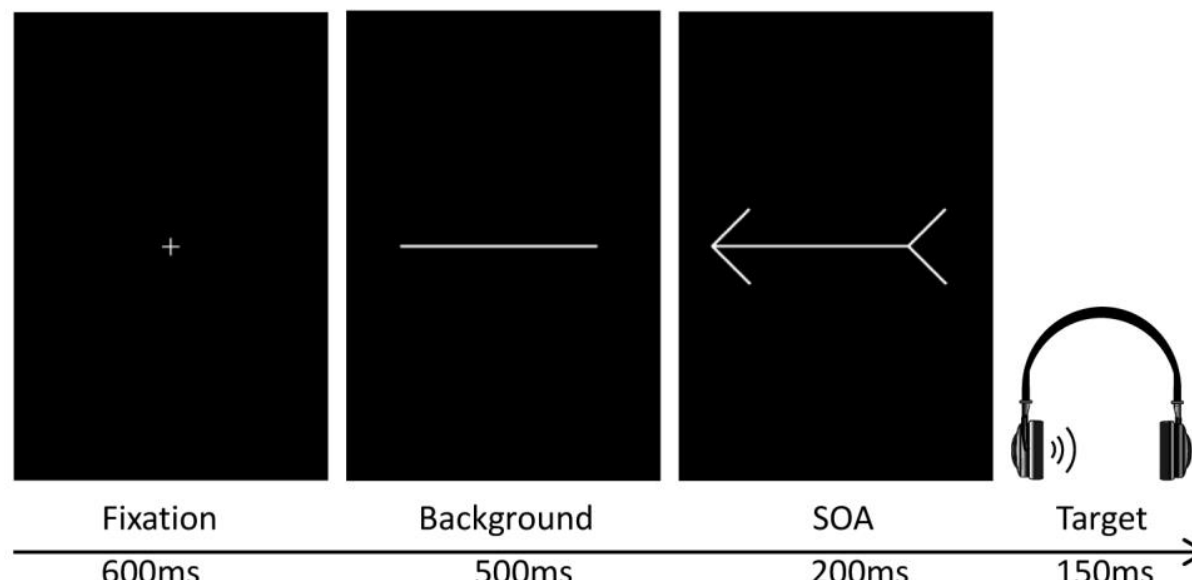

Background

$500 \mathrm{~ms}$

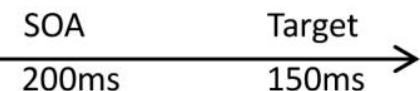

Figure 1. Illustrations of stimulus presentations. Image of the sequence of stimulus presentations is shown. We selected gaze and arrow stimuli as cues. The auditory stimuli were represented by a voice or tone. Speed of response time was measured from the onset of the target in each trial. 
The experiment consisted of eight blocks of 52 trials, including 32 catch trials in which the target did not appear. Forty-eight trials were performed under each condition. Each condition was presented in pseudorandom order. Participants were allowed to rest between blocks. Fifty-two practice trials preceded the experimental trials.

\subsubsection{Date Analysis}

The data were analyzed using SPSS. Incorrect responses (2.1\% of the trials) and responses of $<150 \mathrm{~ms}$ or $>1000 \mathrm{~ms}(0.13 \%$ of the trials $)$ were excluded from the RT analysis. The mean RT difference between invalid and valid under each condition was calculated for each participant. The mean RT difference was analyzed by 2 -way analysis of variance (ANOVA) with Cue conditions (gaze, arrow) and Target conditions (voice, tone) as the within-participant factors.

\subsection{Results and Discussion}

Errors did not show any significant relationship with target conditions, indicating that the participants suffered no speed-accuracy trade-off (all $p>0.05)$ (Table 1).

We explored Validity effects between Cue and Target conditions (Table 1). Thus, we compared the effect between invalid and valid conditions with 2 Cue (gaze, arrow) $\times 2$ Target (voice, tone) mixed-model, repeated measures ANOVA. A 
significant main effect of Target, $F(1,26)=9.864, p<0.01$, partial $\eta^{2}=0.275$ was found, but there were no significant main effects of Cue, $F(1,26)=1.563, p>0.1$. In addition, there was no significant interaction of Cue $\times$ Target $(F(1,26)=0.013, p$ $>0.1$ ) (Figure 2). The results of this experiment showed that the magnitude of cueing effects was not different between gaze and arrow. Importantly, a greater cueing effect was found when target conditions appeared as a voice rather than a tone with both gaze and arrows as cues.

\section{Block design}

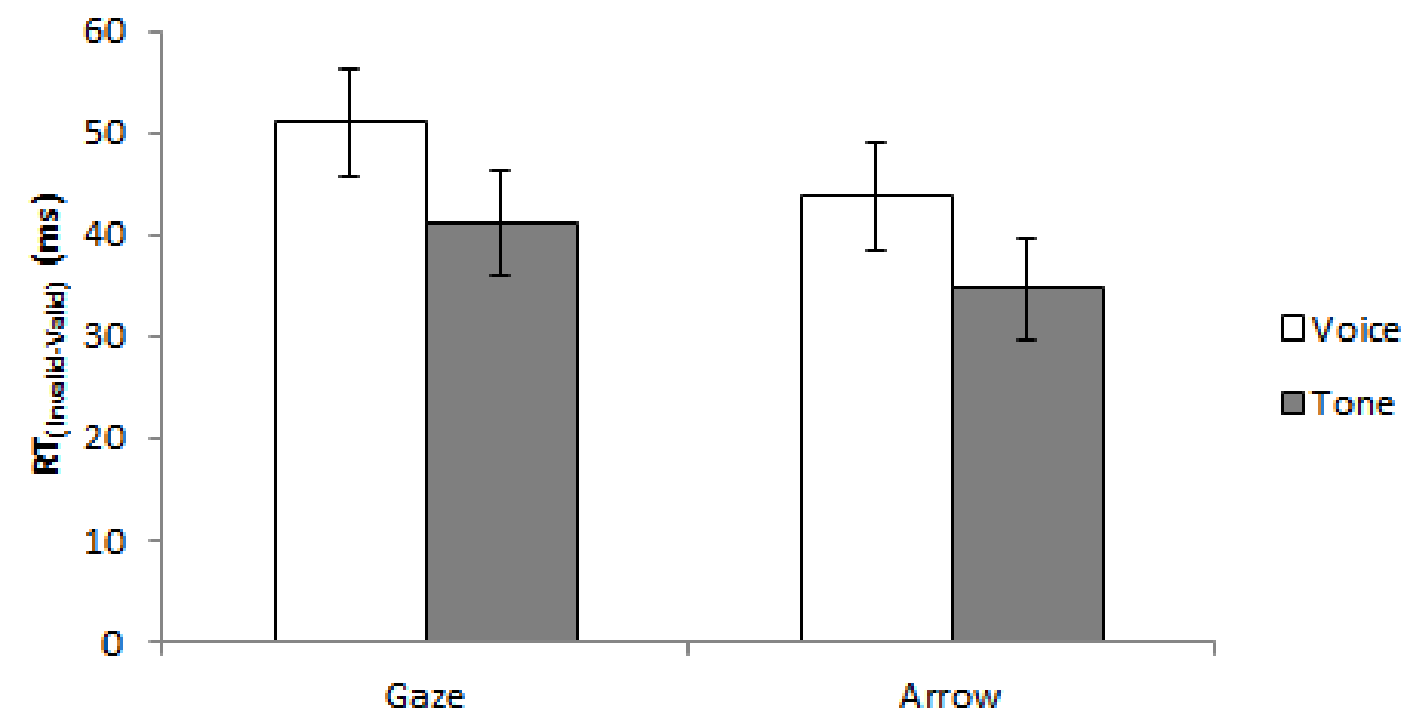

Figure 2. Mean (with SE) RT differences between invalid and valid gaze or arrow

conditions in block conditions. Error bars represent standard errors. 
A previous study (Zhao et al., 2013) proposed that gaze-triggered attention was

enhanced when the cue-target relationship was strong, i.e., social cue and social target.

In contrast, another previous study (Bayliss et al., 2005) found that the attention

orienting effect was enhanced when the target appeared on an interesting target object in

participants with low autistic-like traits. Consistent with this previous study (Bayliss et

al., 2005), the findings of this experiment might support that attention orienting was

mediated by a target object. However, the difference between gaze and arrows might not

be distinguished by the association relationship of cue-target when they are presented

separately in a simple environment. Therefore, in the next experiment we attempted to

investigate the difference in the cueing effect between gaze and arrow under the

unpredictable condition.

\section{Experiment 2}

While Experiment 1 compared the difference between gaze and arrows when

using voice and tone as targets in the block condition, a comparable orienting effect

was found between gaze and arrow. However, the difference in orienting effects

between eye gaze and arrows may be easy to identify in a complex environment.

Birmingham and Kingstone suggested the possibility that key differences between 
social and non-social cues may only become apparent when they are embedded

within a rich environment (Kuhn \& Kingstone, 2009; in a review: Birmingham \& Kingstone, 2009). Hence, a second experiment was conducted, whereby eye gaze and arrows as cues were manipulated in a randomized condition using two sounds as targets, i.e., social voice and non-social tone, to refer the relationship between the cue and target.

\subsection{Materials and Methods}

\subsubsection{Participants}

Twenty-seven naïve participants (mean age $=21.1 \pm 2.37$ SD years; 14 men) took part in the study and received payment. All participants were right-handed and had normal or corrected-to-normal visual and auditory acuity.

3.1.2 Apparatus, design, stimuli, procedure and analysis

All conditions were the same as in Experiment 1A, except that the cueing stimuli of gaze and arrows were manipulated with a randomized condition. Incorrect responses ( $1.7 \%$ of the trials $)$ and responses of $<150 \mathrm{~ms}$ or $>1000 \mathrm{~ms}(0.24 \%$ of the trials $)$ were excluded from the RT analysis. 


\subsection{Results and Discussion}

Errors showed no significant difference between target conditions, indicating that the participants suffered no speed-accuracy trade-off (all $p>0.05$ ) (Table 2).

We explored Validity effects between Cue and Target conditions (Table 2). Then, we compared the effect between invalid and valid conditions with 2 Cue (gaze, arrow) $\times$ 2 Target (voice, tone) mixed-model, repeated measures ANOVA. There were no significant main effects of Cue, $F(1,26)=0.58$ or Target, $F(1,26)=2.074$, both $p>0.1$. A significant interaction of Cue $\times$ Target, $F(1,26)=7.783, p<0.05$, partial $\eta^{2}=0.23$ was found, indicating that the orienting effect was mediated by targets between cues.

The post-hoc test yielded a significantly greater cue effect between gaze and arrows with voice $(p<0.05)$, but not with tone $(p>0.1)$. Additionally, a significantly greater validity effect between voice and tone was found in gaze $(p<0.01)$ but not arrow conditions $(p>0.1)$. Thus, the results showed that attention orienting is enhanced when the associated relationship of cue-target is strong (social gaze as cues and social voice as targets), indicating that the eye gaze cue was preferentially selected to associate with the social stimulus as a target compared to the arrow cue in a complex environment (Figure $3)$. 


\section{Randomized design}

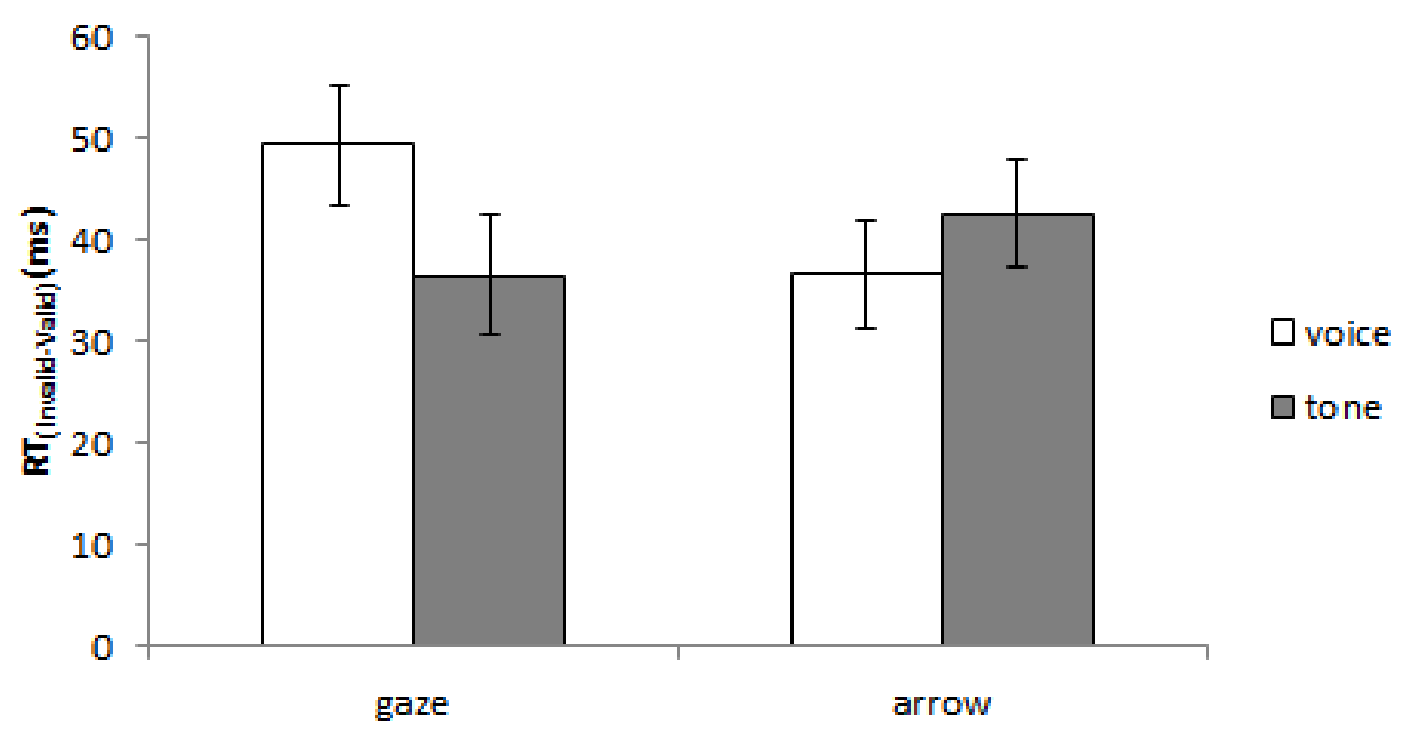

Figure 3. Mean (with SE) RT differences between invalid and valid for gaze and arrow conditions in randomized conditions. Error bars represent standard errors.

Combined Analysis of Experiment 1 and 2

To directly compare these results with Experiment 1, the two cue types of Experiment 2 were entered into a between-subject ANOVA with the Validity effect between invalid and valid conditions. We conducted a 2 Cue (gaze, arrow) $\times 2$ Target (voice, tone $) \times 2$ Groups (Experiment 1 , Experiment 2 ) mixed-model, repeated measures ANOVA (analysis of variance) on these RTs. There were significant main effects of Target $\left(F(1,52)=11.265, p<0.01\right.$, partial $\left.\eta^{2}=0.178\right)$. A significant interaction of Cue $\times$ Target, $F(1,52)=4.787, p<0.05$, partial $\eta^{2}=0.084$ was found, 
indicating that the orienting effect was mediated by targets between cues. More importantly, there was a significant interaction of Cue $\times$ Target $\times$ Groups, $F(1,52)=$ 4.168, $p<0.05$, partial $\eta^{2}=0.074$, suggesting that the orienting effect was different between Experiment 1 and 2.

Post-hoc tests yielded a significantly greater validity effect with voice than tone under gaze $(p<0.05)$ and arrow $(p<0.05)$ conditions in Experiment 1 , indicating a comparable orienting effect between gaze and arrows as cues. However, no significant Target difference was found with arrows $(p>0.1)$ in Experiment 2, although there was a significant Target difference between voice and tone with gaze ( $p<0.01$ ), indicating a strong relationship between the gaze cue and voice target compared to the tone target in attention orienting. Additionally, we found a significantly greater cue effect between gaze and arrows with voice $(p<0.05)$ in Experiment 2, indicating that the strong relationship between the gaze cue and voice target enhanced attention orienting. Eye gaze as a cue was preferentially selected to associate with voice as a target compared with arrows as a cue. Thus, these results showed that the orienting strategy was flexibly mediated to respond to environmental changes between Experiment 1 and 2. 


\section{General discussion}

The present study investigated the effect of attention orienting by sound targets (i.e., voice and tone) between gaze and arrows in simple and unpredictable conditions.

Given the results of Experiment 1 and 2, attention orienting was influenced by the associated relationship between cue and target. Particularly in the unpredictable condition, the enhanced cueing effect to the voice target was found only in the gaze cue condition. This result suggests that the difference in attention orienting between gaze and arrows as cues might become apparent when they are implemented randomly in an unpredictable condition.

The results of Experiment 1 found that attention orienting was enhanced by a voice versus tone target with both gaze and arrow cues, indicating that there was a comparable effect on orienting between gaze and arrow cues. A previous study found greater gaze-triggered orienting when using voice as the target rather than tone (Zhao et al., 2013). The present study extended the effect of auditory targets on attention orienting by another kind of cue (i.e. arrow). Another previous study (Bayliss \& Tipper, 2005) also showed a comparable orienting effect between gaze and arrow cues, and greater orienting of attention was found when the target appeared on a face rather than a scrambled face in individuals with low autistic-like traits, but an 
opposite enhanced effect was found in individuals with high autistic-like traits. The study suggested that the use of central non-predictive cues can produce target object attentional effects to effectively capture important information, indicating that this is seemingly due to the difference in target object interest. Particularly in individuals with high autistic-like traits, the results were thought to be influenced by good attention to detail. In contrast, typically developing individuals commonly appear to be hard-wired to attend to faces more quickly and powerfully than other stimuli (Calder, 2011). Faces provide a wealth of information about others and therefore attract more interest. Therefore, this might explain the results of the enhanced orienting effect when the target appeared on a face in individuals with low autistic-like traits. Consistent with this study, the results of Experiment 1 might also support that attention orienting was mediated by a difference in target object interest. As with a face stimulus, the voice is a social stimulus, commonly called the “Auditory Face," and plays an important role in social cognition (Belin, Zatorre, Lafaille, Ahad, \& Pike, 2000). Thus, in the current study, we suggest that participants might have been automatically biased toward an interesting stimulus, such as a face or voice, having a greater effect on attention orienting. 
However, in the unpredictable condition, attention orienting was found to be influenced by the associated relationship between cue-target, rather than only being influenced by target objects. In a previous study (Zhao et al., 2013), we proposed that attention orienting was enhanced when the associated cue-target relationship was strong, such as when both the cue and target had a congruent meaning. Consistent with this, the results of Experiment 2 might show that the enhanced gaze-cueing effect of a voice target emerged from the strong relationship between the cue and target, i.e., using social gaze as the cue and social voice as the target. Bayliss, Schuch, \& Tipper (2010) demonstrated that the gaze direction of happy faces more effectively oriented their attention to pleasant targets than disgusted faces. Kuhn \& Tipples (2011) also found that fearful faces showed a more enhanced gaze cueing effect than happy faces only when searching for a threatening target. These studies also suggested that the congruency of the meaning between cue and target has a critical role in orienting attention. Given that social gaze and non-social arrow cues were implemented in the same block, the congruency of the meaning between a social cue (i.e., gaze) and a social target (i.e., voice) is obvious in a randomized design compared to a blocked design. Thus, a strong cueing effect might emerge when a gaze cue and a voice target are presented and inhibited when an arrow cue and a voice target are presented. 
Importantly, the effect of attention orienting was found to differ between gaze and arrows as cues, although previous studies have reported that eye gaze is not a unique attention-triggering stimulus (Bayliss \& Tipper, 2005; Borjon et al., 2011; Kuhn \& Kingstone, 2009; Tipples, 2002). We found that the attention-orienting effect was clearly enhanced when eye gaze was preferentially selected to associate with a target with a congruent meaning compared to an arrow in an unpredictable condition, although a comparable effect was induced in a simple condition. The difference between gaze and arrow was not affected by target detection difficulties in simple and unpredictable conditions (two 3-way repeated measures ANOVA (Cue $\times$ Target $\times$ Validity) was performed, but there was no significant main effect of Target $(F(1,26)=0.222, p=$ $0.641)$ in Experiment 1 and Target $(F(1,26)=1.213, p=0.281)$ in Experiment 2$)$. Previous studies also demonstrated a difference in attention orienting between gaze and arrows in complex experimental paradigms (Friesen et al., 2004; Marotta, Lupiáñez, \& Casagrande, 2012; Marotta, Lupiáñez, Martella, et al., 2012). However, most of the research investigating the difference between gaze and arrows focused on whether it triggers a shift in attention orienting, while the impact of the associated relationship between cue and target has been ignored. It also seems that the influence of environmental changes in attention orienting has been overlooked. The findings in the 
current study might give a new perspective to explore differences in attention orienting between eye gaze and arrows. Consistent with the idea that the unique nature of gaze becomes apparent in a rich environment (Kuhn \& Kingstone, 2009 and Birmingham \& Kingstone, 2009), our findings suggest that attention orienting is obviously different between gaze and arrows in the unpredictable condition, which seems closer to a real environment than the simple condition.

Clearly, the current study suggests that a different strategy of orienting is employed between simple and rich environments. Given the environmental variability in a natural setting, how to rapidly and effectively search for important information might play a critical role in human evolution. In the simple condition, only one type of cueing was presented as a cue, therefore the importance could not be compared directly between gaze and arrow as cues. However, in the unpredictable condition, participants might preferentially process a more important social cue (i.e. gaze) than a non-social cue (i.e. arrow) in attention orienting. In addition, from an evolutionary point of view, people can rapidly avoid danger if they are able to flexibly utilize their attention in rich and hazardous environments, such as when living in virgin forest. Consistent with this view, our findings might also reflect that participants employ a flexible orienting strategy to 
better respond to environment changes, and therefore to effectively capture important information.

The current results have implications for social orienting in the development of social cognition. Social orienting is the need to flexibly modulate our attention strategy in real life. Impaired social orienting (e.g., another's eye gaze, gestures etc.) in real life has been well documented in Autism Spectrum Disorder (ASD) (American Psychiatric Association [APA], 2000). In contrast, experimental studies generally have reported intact social orienting in ASD (Chawarska, Klin, \& Volkmar, 2003; Kylliäinen \& Hietanen, 2004; Rutherford \& Krysko, 2008; Senju, Tojo, Dairoku, \& Hasegawa, 2004; Swettenham, Condie, Campbell, Milne, \& Coleman, 2003; Vlamings, Stauder, Son, \& Mottron, 2005); for a review (Nation \& Penny, 2008), although the impairment in attention triggered by social gaze cues has been observed in individuals with ASD (Goldberg et al., 2008; Marotta et al., 2013; Ristic et al., 2005). However, these studies only investigated social orienting in a simple environment, such as Experiment 1 in the present study. Given the environmental complexity in real life, this study raises the question of whether individuals with ASD show impairment of gaze-triggered attention in environments in which a flexible attentional strategy for environmental changes is required. 
In summary, the findings of this study give a new perspective to explain the

difference in attention orienting between eye gaze and arrows. The difference in

attention orienting between gaze and arrows became apparent in the unpredictable

condition, which seemed closer to a real environment than the simple condition.

Attention orienting is mediated by the associated cue-target relationship and therefore

enables a better flexible response to environmental changes.

\section{References}

American Psychiatric Association[APA]. (2000). Diagnostic and statistical manual for mental disorders (DSM-IV-TR). Washington, DC: APA.

Baron-Cohen, S. (1995). Mindblindness: An Essay on Autism and "theory of Mind": Bradford Books.

Bayliss, A. P., \& Tipper, S. P. (2005). Gaze and arrow cueing of attention reveals individual differences along the autism spectrum as a function of target context. British Journal of Psychology, 96(1), 95-114.

Bayliss, A. P., Schuch, S., \& Tipper, S. P. (2010). Gaze cueing elicited by emotional faces is influenced by affective context. Visual Cognition, 18, 1214-1232.

Birmingham, E., \& Kingstone, A. (2009). Human social attention. Progress in Brain Research, 176, 309-320.

Borjon, J. I., Shepherd, S. V., Todorov, A., \& Ghazanfar, A. A. (2011). Eye-gaze and arrow cues influence elementary sound perception. Proceedings of the Royal Society B: Biological Sciences, 278(1714), 1997-2004.

Calder, A. (2011). Oxford Handbook of Face Perception: OUP Oxford.

Belin, P., R.J. Zatorre, P. Lafaille, P. Ahad, B. Pike (2000). Voice-selective areas in human auditory cortex. Nature, 403(6767), 309-312.

Chawarska, K., Klin, A., \& Volkmar, F. (2003). Automatic Attention Cueing Through Eye Movement in 2-Year-Old Children With Autism. Child Development, 74(4), 1108-1122.

Ekman, P., \& Friesen, W. V. (1976). Pictures of facial affect. Palo Alto, CA: Consulting Psychologists Press.

Friesen, C. K., \& Kingstone, A. (1998). The eyes have it! Reflexive orienting is triggered by nonpredictive gaze. Psychonomic Bulletin \& Review, 5(3), 490-495. 
Friesen, C. K., Ristic, J., \& Kingstone, A. (2004). Attentional Effects of Counterpredictive Gaze and Arrow Cues. Journal of Experimental Psychology: Human Perception and Performance, 30(2), 319-329.

Goldberg, M., Mostow, A., Vecera, S., Larson, J. G., Mostofsky, S., Mahone, E. M., et al. (2008). Evidence for Impairments in Using Static Line Drawings of Eye Gaze Cues to Orient Visual-Spatial Attention in Children with High Functioning Autism. Journal of Autism and Developmental Disorders, 38(8), 1405-1413.

Kryzak, L. A., Bauer, S., Jones, E. A., \& Sturmey, P. (2013). Increasing responding to others' joint attention directives using circumscribed interests. Journal of Applied Behavior Analysis, 46(3), 674-679.

Kuhn, G., \& Kingstone, A. (2009). Look away! Eyes and arrows engage oculomotor responses automatically. Attention, Perception, \& Psychophysics, 71(2), 314-327.

Kylliäinen, A., \& Hietanen, J. K. (2004). Attention orienting by another's gaze direction in children with autism. Journal of Child Psychology and Psychiatry, 45(3), 435-444.

Marotta, A., Lupiáñez, J., \& Casagrande, M. (2012). Investigating hemispheric lateralization of reflexive attention to gaze and arrow cues. Brain and Cognition, 80(3), 361-366.

Marotta, A., Lupiáñez, J., Martella, D., \& Casagrande, M. (2012). Eye gaze versus arrows as spatial cues: Two qualitatively different modes of attentional selection. Journal of Experimental Psychology: Human Perception and Performance, 38(2), 326-335.

Marotta, A., Pasini, A., Ruggiero, S., Maccari, L., Rosa, C., Lupiáñez, J., et al. (2013). Inhibition of Return in Response to Eye Gaze and Peripheral Cues in Young People with Asperger's Syndrome. Journal of Autism and Developmental Disorders, 43(4), 917-923.

Naoi, N., Tsuchiya, R., Yamamoto, J.-I., \& Nakamura, K. (2008). Functional training for initiating joint attention in children with autism. Research in Developmental Disabilities, 29(6), 595-609.

Nation, K., \& Penny, S. (2008). Sensitivity to eye gaze in autism: Is it normal? Is it automatic? Is it social? Development and Psychopathology, 20(1), 79-97.

Oldfield, R. C. (1971). The assessment and analysis of handedness: The Edinburgh inventory. Neuropsychologia, 9(1), 97-113.

Posner, M. (1980). Orienting of attention. The Quarterly Journal of Experimental Psychologyl, 32, 3 - 25.

Ristic, J., Mottron, L., Friesen, C. K., larocci, G., Burack, J. A., \& Kingstone, A. (2005). Eyes are special but not for everyone: The case of autism. Cognitive Brain Research, 24(3), 715-718.

Rutherford, M. D., \& Krysko, K. M. (2008). Eye Direction, Not Movement Direction, Predicts Attention Shifts in Those with Autism Spectrum Disorders. Journal of Autism and Developmental Disorders, 38(10), 1958-1965.

Senju, A., Tojo, Y., Dairoku, H., \& Hasegawa, T. (2004). Reflexive orienting in response to eye gaze and an arrow in children with and without autism. Journal of Child Psychology and Psychiatry, 45(3), 445-458. 
Swettenham, J., Condie, S., Campbell, R., Milne, E., \& Coleman, M. (2003). Does the perception of moving eyes trigger reflexive visual orienting in autism? Philosophical Transactions of the Royal Society B: Biological Sciences, 358(1430), 325-334.

Tipples, J. (2002). Eye gaze is not unique: Automatic orienting in response to uninformative arrows. Psychonomic Bulletin \& Review, 9(2), 314-318.

Vlamings, P. H. J. M., Stauder, J. E. A., Son, I. A. M., \& Mottron, a. L. (2005). Atypical Visual Orienting to Gaze- and Arrow-Cues in Adults with High Functioning Autism. Journal of Autism and Developmental Disorders, 35(3), 267-277.

Zhao, S., Uono, S., Yoshimura, S., Kubota, Y., \& Toichi, M. (2013). Can gaze-cueing be helpful for detecting sound in autism spectrum disorder? Research in Autism Spectrum Disorders, 7(10), 1250-1256. 